\title{
Turn Performance of an Air-Breathing Hypersonic Vehicle
}

\author{
Derek J. Dalle*, Sean M. Torrez ${ }^{\dagger}$, and James F. Driscoll ${ }^{\ddagger}$ \\ University of Michigan, Ann Arbor, MI 48109
}

\begin{abstract}
Turning flight of an X-43-like air-breathing hypersonic vehicle is discussed. Equations of motion for turning flight of an air-breathing hypersonic vehicle are derived, and several constant-altitude turning flight configurations are analyzed. In addition to calculating an operating map for a combination of altitudes and Mach numbers, a linearization is performed for the case of a $2 g$ turn, and stability properties are discussed. The stability properties are compared to those of a non-turning flight condition at the same altitude and Mach number. A low-order vehicle model, which includes effects due to wave interactions, fuel mixing, and other factors, is used for the calculation.
\end{abstract}

Nomenclature

\begin{tabular}{|c|c|c|c|}
\hline $\mathbf{C}$ & transformation matrix & $\theta$ & $=$ pitch angle \\
\hline$e$ & eccentricity & $\lambda$ & longitude \\
\hline e & column of the identity matrix & $\xi$ & independent trim variables \\
\hline f & specific force vector & $v$ & dependent trim variables \\
\hline $\mathbf{F}$ & generic vector function & $\phi$ & roll angle \\
\hline$g$ & $=9.80665 \mathrm{~m} / \mathrm{s}^{2}$ & $\chi$ & velocity heading angle \\
\hline g & acceleration vector due to gravity & $\psi$ & body axis heading angle \\
\hline$h$ & geodetic altitude & $\omega$ & angular rate \\
\hline $\mathbf{I}$ & specific inertia tensor & $\omega$ & angular velocity vector \\
\hline$L$ & geodetic latitude & & \\
\hline$M$ & $=$ Mach number & \multicolumn{2}{|c|}{ subscript/superscript frame labels } \\
\hline$n$ & load factor & $b$ & $=$ body frame \\
\hline M & specific moment or torque vector & $e$ & $=$ Earth-centered, Earth-fixed frame \\
\hline$p$ & roll rate & $i$ & $=$ Earth-centered inertial frame \\
\hline$q$ & pitch rate & $n$ & $=$ navigation frame \\
\hline$Q$ & $=$ dynamic pressure & & \\
\hline$r$ & $=$ yaw rate & \multicolumn{2}{|c|}{ subscripts } \\
\hline $\mathbf{r}$ & position vector & $C E$ & collective elevator angle \\
\hline$R$ & radius or distance & $C R$ & $=$ collective rudder angle \\
\hline $\mathbf{u}$ & vector of control variables & $D$ & downward component \\
\hline$u$ & velocity scalar & $D E$ & $=$ difference between elevators \\
\hline $\mathbf{v}$ & velocity vector & $D R$ & $=$ difference between rudders \\
\hline$v$ & velocity scalar & $E$ & east component \\
\hline$V$ & velocity magnitude & $E R$ & $=$ fuel-air equivalence ratio \\
\hline$w$ & velocity scalar & $j$ & $=$ vector component index \\
\hline$x$ & reference axis or coordinate & $k$ & $=$ vector component index \\
\hline $\mathbf{x}$ & vector of state variables & $N$ & $=$ north component \\
\hline$y$ & reference axis or coordinate & $Q$ & value at equator \\
\hline $\mathbf{y}$ & vector of output variables & & \\
\hline$z$ & $=$ reference axis or coordinate & \multicolumn{2}{|c|}{ modifiers } \\
\hline$\alpha$ & angle of attack & $\dot{a}$ & time derivative \\
\hline$\beta$ & $=$ sideslip angle & $\ddot{a}$ & second time derivative \\
\hline$\gamma$ & $=$ flight path angle & $\bar{a}$ & value at trim \\
\hline$\delta$ & deflection angle & $\Delta a$ & small perturbation \\
\hline$\eta$ & $=$ small step size & $a^{T}$ & matrix transpose \\
\hline
\end{tabular}

\footnotetext{
* Graduate Research Assistant, Department of Aerospace Engineering, AIAA Member, dal le@umich .edu

${ }^{\dagger}$ Graduate Research Assistant, Department of Aerospace Engineering, AIAA Member, smtorrez@umich. edu

$\ddagger$ Professor, Department of Aerospace Engineering, AIAA Fellow, jamesfd@umich . edu
} 


\section{Introduction}

In addition to maximizing fuel efficiency, ensuring adequate thrust and lift, and limiting heat transfer, a hypersonic vehicle must be able to balance all of the forces acting on it such that the velocity and attitude of the vehicle remain constant. Using this as a guideline, we define the flight envelope as the set of flight Mach numbers, $M$, and altitudes, $h$, at which the vehicle is able to meet these conditions for steady, level flight. In order to simulate this balanced, or trimmed, flight condition, the forces on the vehicle must be calculated for many combinations of input variables, and a model is required that can evaluate the performance of the entire vehicle in a few seconds or less.

Previous models capable of analyzing trim of a full air-breathing hypersonic vehicle include three-degree-offreedom longitudinal models by Schöttle and Hillesheimer [1], Chavez and Schmidt [2], Bolender and Doman [3], and Parker et al. [4], and a six-degree-of-freedom model by Frendreis and Cesnik [5]. Recent work by the authors [6] discussed trim analysis using more accurate vehicle modeling. For example, we now account for complex phenomena such as wave interactions, finite-rate chemistry, and fuel mixing. Simpler models may be accurate for a narrow range of conditions at which a good vehicle design can ensure proper compression and fuel mixing, but factors such as wave interactions and poor fuel mixing have been shown to play a significant role for a vehicle with a wide flight envelope $[7,8]$.

The present model, known as Michigan/AFRL Scramjet Trim, or MASTrim, incorporates a three-dimensional vehicle geometry and includes a higher-fidelity one-dimensional model of the propulsive system than what was previously used. Although it lacks a model for vehicle deformations, we can accommodate the effects of flexibility by superimposing the mode shapes of the vehicle onto the geometry. We used version 2.2 of this code to obtain the data in this paper. The code is designed for analysis of vehicles that are designed with planar inlets-such as the X-43, X-51, and the National Aerospace Plane. An example vehicle is shown in Fig. 1, which is the vehicle used in the present analysis. The package also includes a tool to produce similar vehicles based on 62 design parameters. Example parameters include vehicle length, stabilator location, and engine width.

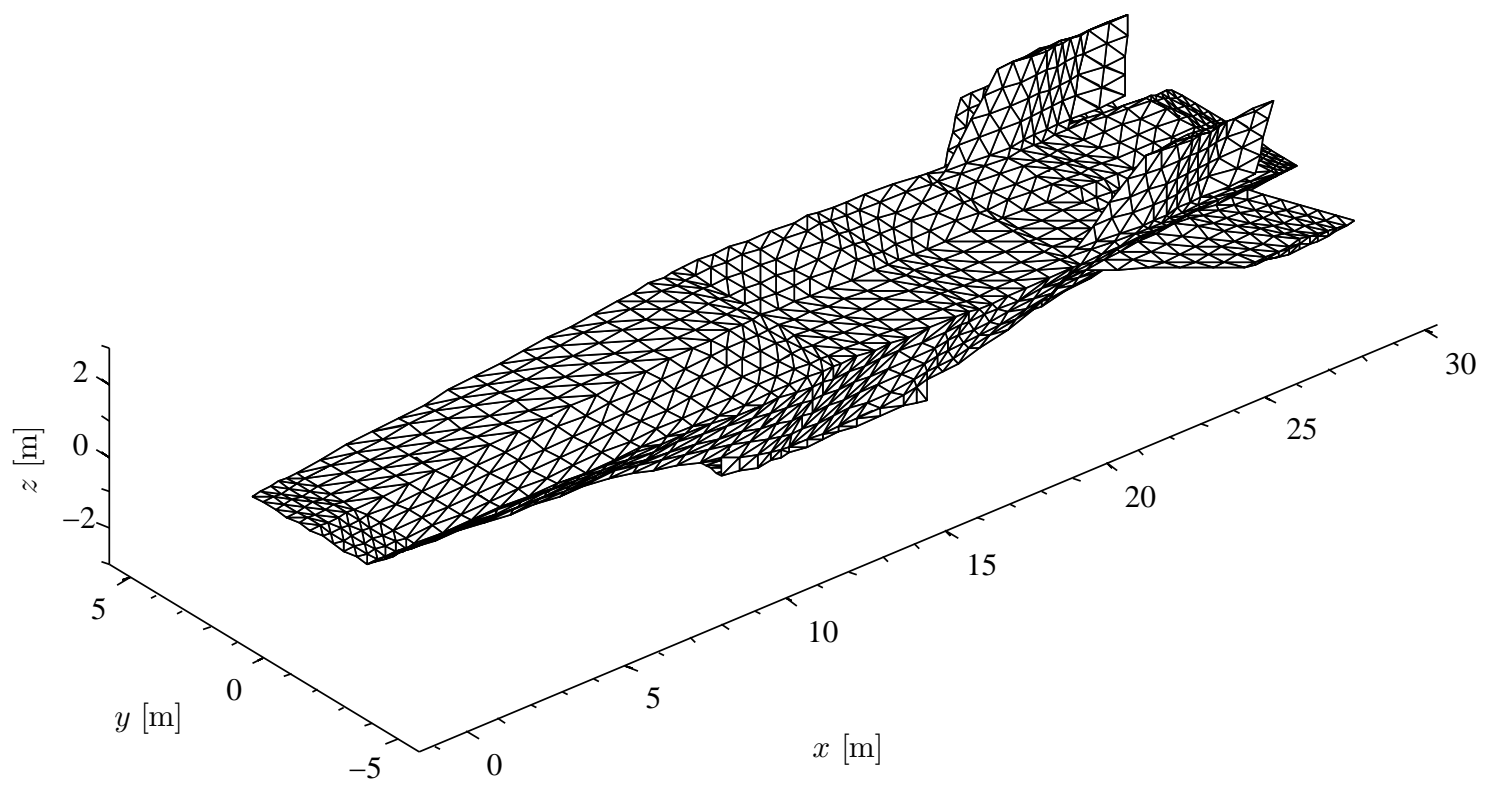

Figure 1. Isometric view of example vehicle.

In our view, the advantage to using this type of model is that it has the capability to model the performance of a hypersonic vehicle over a wide range of conditions with relatively little computation. For example, previous work, such as [7,9], has shown that a very small change in angle of attack or Mach number can on some occasions cause a large change in the inlet performance. An inlet model that does not account for wave interactions would have no way to predict this performance drop. It is possible to design inlets that reduce this effect [10] and as a result a simplified analysis may be accurate for initial analysis of on-design performance. However, a more capable model is needed to develop such an inlet design. 
Turning flight for hypersonic vehicles has been studied in detail in the past, particularly as it applies to reentering spacecraft $[11,12,13]$. The dependence of performance on flight condition adds considerably more complexity to the air-breathing version of hypersonic turning. In this realm, Mooij [14] developed and documented the performance of an algorithm to control the heading angle and bank angle of a hypersonic vehicle, but the reference trajectory was not for turning flight. Schöttle and Hillesheimer [1] considered trajectories that included turning, but a very simple model was used. The most relevant work was done by Raney and Lallman [15] who considered a control law for a hypersonic vehicle trajectory with several different phases, including a change of heading.

The goal of this paper is to combine detailed turn performance with the more sophisticated propulsion model provided by MASTrim. Chavez and Schmidt [2], Tarpley and Lewis [16], Parker et al. [4], Bolender and Doman [3], and others have all done work on stability analysis of hypersonic vehicles. This work will supplement theirs by adding turning flight about a rotating, ellipsoidal Earth to the flight conditions for which open-loop stability analysis is available.

We use a rotating, elliptical model for Earth, which means that the trimmed flight condition is dependent on latitude and heading angle. In this analysis we investigate how the operating map is affected by a number of parameters. Specifically, the conditions that are varied are Mach number, $M$; altitude, $h$; load factor, $n$; and dynamic pressure, $Q$.

\section{Hypersonic Vehicle Model}

The vehicle model consists of separate models for the inlet, isolator, combustor, nozzle, and the external aerodynamic components that are carefully integrated to ensure that the flight dynamics of the vehicle are realistically captured. For modeling purposes, the vehicle is split into propulsive and nonpropulsive sections, which is consistent with standard convention. The engine model is responsible for the inlet, isolator, combustor, and nozzle, and the external aerodynamic model is responsible for the remaining surfaces. In Fig. 2, the surfaces that are considered to be part of the engine model are shown in red. This modeling approach is limited to vehicles that are similar to an X-43. More precisely, it is limited to vehicles for which the inlet and nozzle can be approximated reasonably as two-dimensional flow, but even for these vehicles the model neglects three-dimensional flow effects such as lateral spillage.

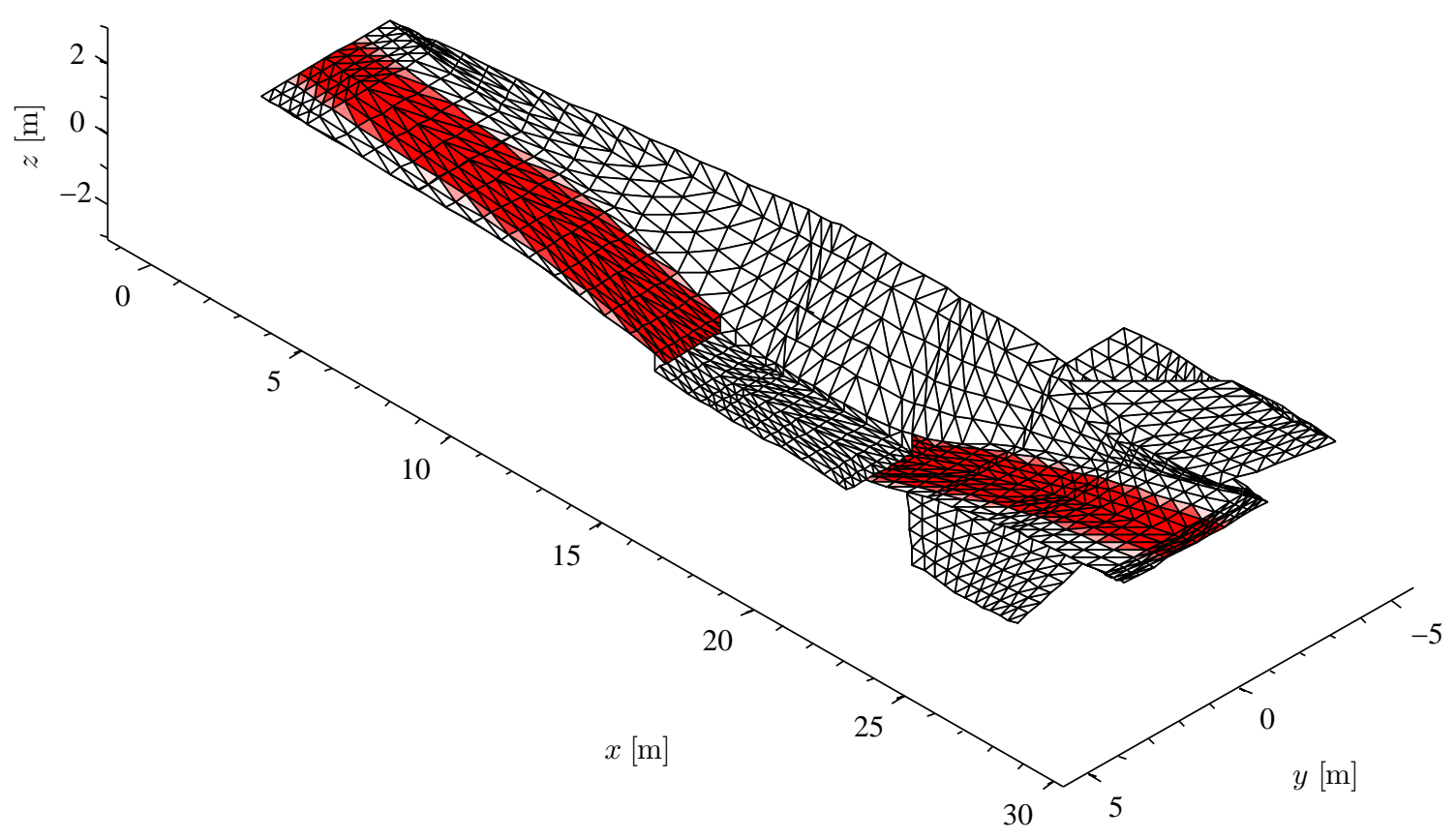

Figure 2. View of the vehicle from below with propulsive surfaces in red. 


\section{A. Engine Model}

The engine model is the primary component of MASTrim, and as a result, it has its own acronym. We refer to the engine model as the Michigan/AFRL Scramjet In Vehicle, or MASIV, code. The MASIV engine model is designed to have enough fidelity to capture the important physics of the combustion process while still being able to run in a few seconds or less on a single personal computer. In a true reduced-order model approach, a large amount of computationally expensive analysis is needed for each new vehicle design, and afterward analysis can be done rapidly [17]. Rather than taking this kind of approach, we have developed the model as a direct, low-order physics model. As a result, the MASIV model can be used to design a new vehicle geometry in just a few seconds, and the first trimmed flight condition can be calculated in only one or two minutes after that.

Of course, speed is not the only concern in the MASIV model. Actually, the primary contribution that the model provides is a higher level of complexity and fidelity than what has been previously available for control or flight dynamics studies during the conceptual design phase of the vehicle design process. For example, we found that accounting for interactions between shock waves in the inlet affects the performance by a substantial amount for offdesign flight conditions [7]. As a result, we developed a method to design inlets that is more robust with respect to a wider range of flight conditions [10]. In another example, we found that inadequate fuel-air mixing in the combustor caused a dramatic decrease in fuel efficiency [8].

The inlet and nozzle use the same underlying physics to predict performance. This model is called Supersonic Aerodynamic Model Using Riemann Interactions (SAMURI), and it creates a solution that is similar in appearance to two-dimensional computational fluid dynamics (CFD). Instead of creating a grid and solving for the flow conditions at each point in the grid, SAMURI works by tracking the positions of the vehicle surfaces, shock waves, expansion waves, and contact discontinuities. It begins at the most upstream coordinate in the flow region and only computes wave and post-wave properties at leading edges, vertices of the vehicle surface, points where at least two waves interact, or points where at least one wave interacts with the vehicle surface. This approach is limited to two-dimensional surfaces that consist of a series of flat plates, although a curved surface potentially can be modeled in this way by splitting the curve into a finite number of flat sections. Also, since this approach assumes that information cannot propagate upstream, it is limited to supersonic flows. The output of this model is a collection of regions for which the boundaries are all either waves or the surface of the vehicle itself. In each of these regions, the flow properties (pressure, temperature, etc.) are constant.

After the waves and flow solution have been computed, net forces are calculated using a control volume analysis. This requires tracing streamlines (upstream in the case of the inlet and downstream in the case of the nozzle). Viscous forces are taken into account in the form of a boundary layer, which is modeled as a momentum thickness. The flow in the boundary layer has a thermodynamic state (pressure, density, temperature) but no flow velocity. The height of the momentum layer is defined such that a control volume analysis predicts drag using these assumptions in a way that is consistent with conservation of mass, momentum, and energy [18]. The actual value of the momentum thickness at each point is calculated using the Van Driest II method [19], which is commonly used in the part of the design process where this code is intended to apply. An example solution is shown in Fig. 3.

The engine flowpath consists of an inlet, isolator, combustor, and nozzle. The isolator has a constant area and is described in [20] while the combustor consists of a constant-area section followed by a diverging section and is described in $[20,21]$.

The combustion code marches the one-dimensional flow conservation equations from the beginning of the combustor to the end of the internal nozzle. All flow states are allowed to vary in the downstream axial direction. To keep the model one-dimensional, only derivatives with respect to the axial coordinate are considered. Some quantities, such as jet spreading and mixing, vary in the transverse directions, but they may only vary algebraically such that their evolutions do not depend on the information propagating downstream. This allows, to some degree, for a model of fuel-air mixing without introducing the computational expense required for a two- or three-dimensional flow solution.

When the engine is operating in scram mode, the flow velocity is higher than the local speed of sound throughout the combustor. However, the heat release in the combustor tends to drive the local Mach number to 1, and if enough fuel is added, the flow through the combustor eventually becomes subsonic (thermally choked) at some point. When this happens, the entire combustor switches to ram mode, and the operation changes significantly. Although we have developed a model for ram-mode flight, it has not been tested adequately for inclusion in this paper, and the results in this discussion are within the scram-mode flight envelope.

The result of this analysis is a one-dimensional profile for density, pressure, temperature, flow velocity, and species mass fractions. These results are shown in Fig. 4 for trimmed flight at Mach 8 and an altitude of $26 \mathrm{~km}$. Using this data, we calculate the net forces and moments on the combustor and isolator. Actually, the forces and moments are calculated per unit width since the engine model is two-dimensional. The force on the engine is then the sum of the forces on each component times the width of the engine. 


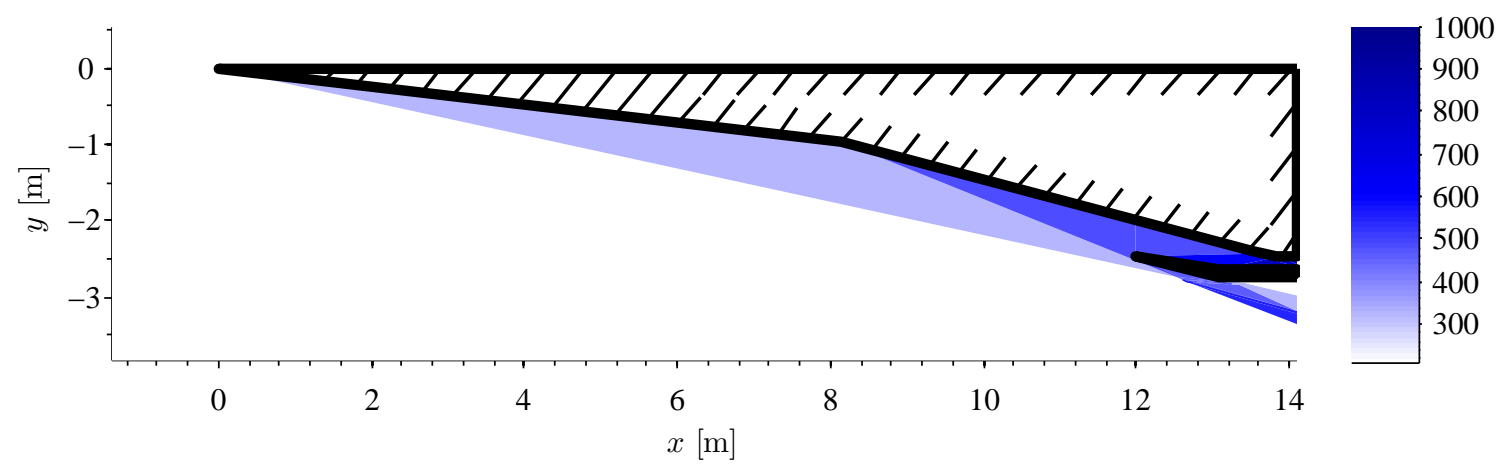

a) Full inlet.

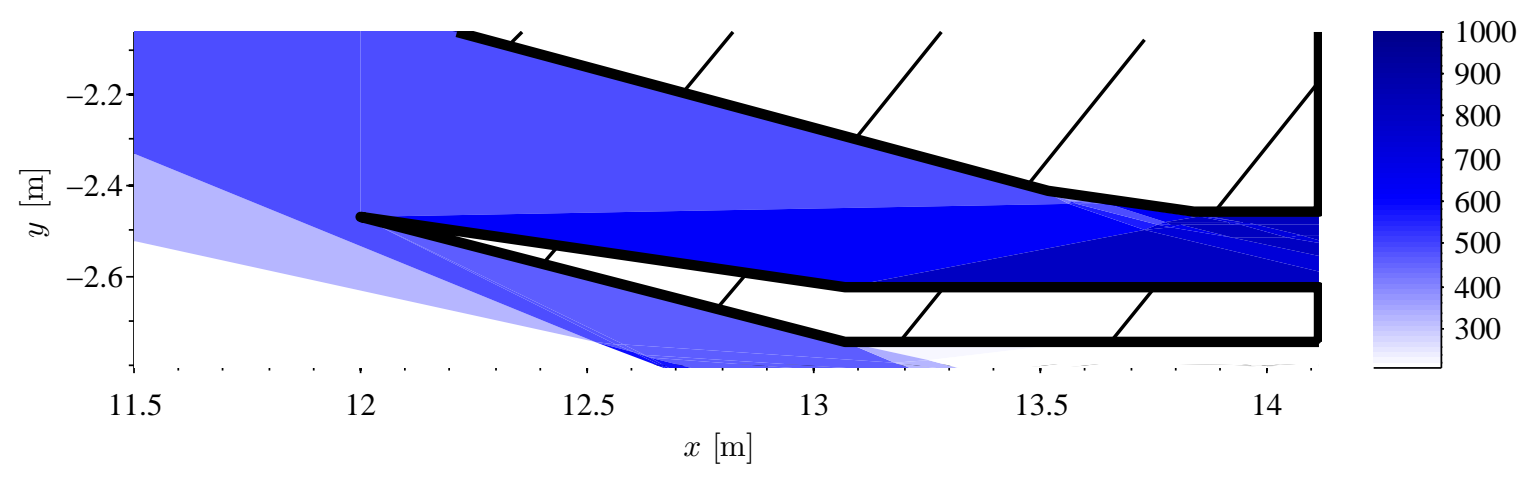

b) Cowl region.

Figure 3. Temperature plot for an example inlet for trimmed flight at Mach 8 and an altitude of $26 \mathrm{~km}$.

\section{B. External Aerodynamics}

The remaining surfaces (those that are not red in Fig. 2) are handled by a simple shock/expansion method with a boundary layer correction to account for viscous drag. For each small triangular surface, the freestream velocity is projected onto the surface. The angle between the freestream and this projected vector is then used to compute the thermodynamic properties behind either a shock or a Prandtl-Meyer expansion. The projected vector is also used to compute the boundary layer properties using the Van Driest II method [19].

For any surface that is not along a leading edge, a boundary layer already exists on the upstream surfaces. Considering the boundary layer to start over on each surface would result in a considerable overestimate of viscous drag. To account for this, a momentum thickness is calculated for each edge. For each surface, the momentum thickness at the upstream edge(s) is used to calculate the momentum thickness at the downstream edge(s), which is then passed on to the next surface. This model provides a first estimate of the viscous drag on the exterior of the vehicle, but it does not account for shock-boundary layer interactions. This differs from the boundary layer model used in the inlet and nozzle, in which the deflection angle caused by the boundary layer itself is considered part of the deflection angle that determines the strength of the shock.

\section{Equations of Motion}

For hypersonic vehicles, the velocities and altitude are high enough that the assumption a flat Earth is no longer valid. As a result, we employ a model that is consistent with a rotating WGS84 Earth [22, 23]. For example, an aircraft flying east at a constant altitude above the equator would require a lift force that is about $13 \%$ less than what would be predicted when using the flat-Earth equations of motion.

The output of the hypersonic vehicle model is a net specific force (i.e. net force divided by vehicle mass) and net 


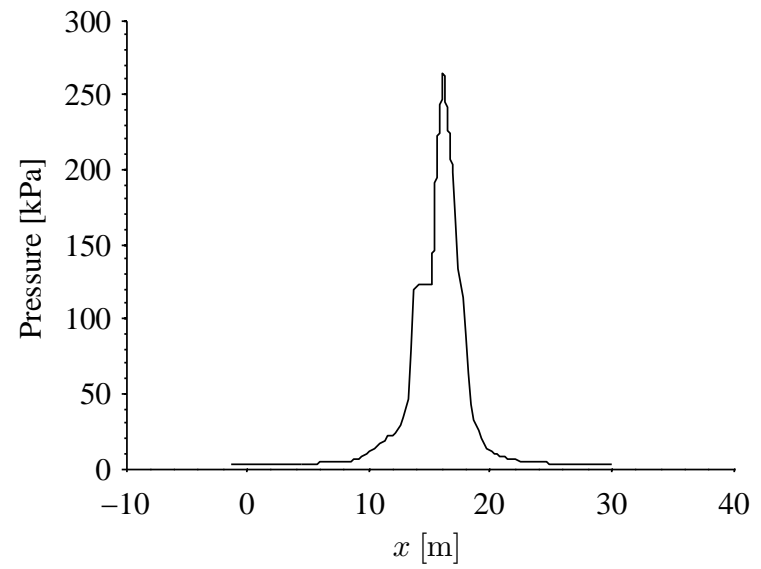

a) Pressure.

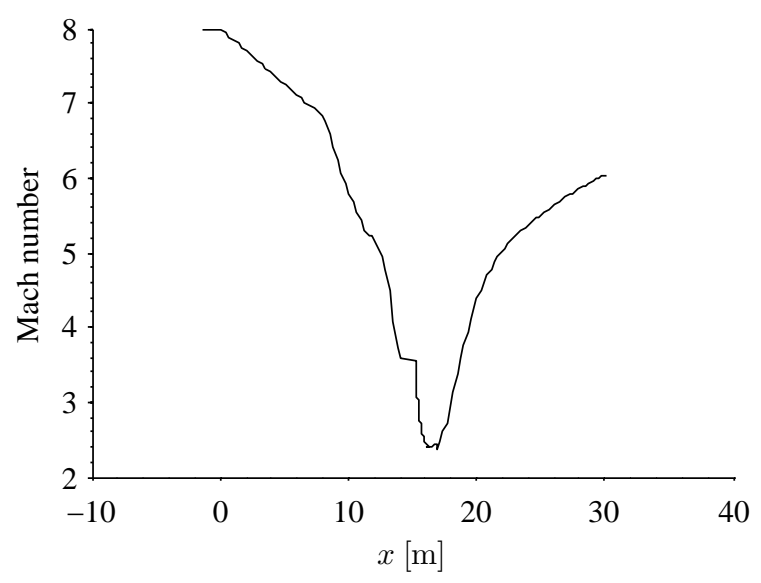

c) Mach number.

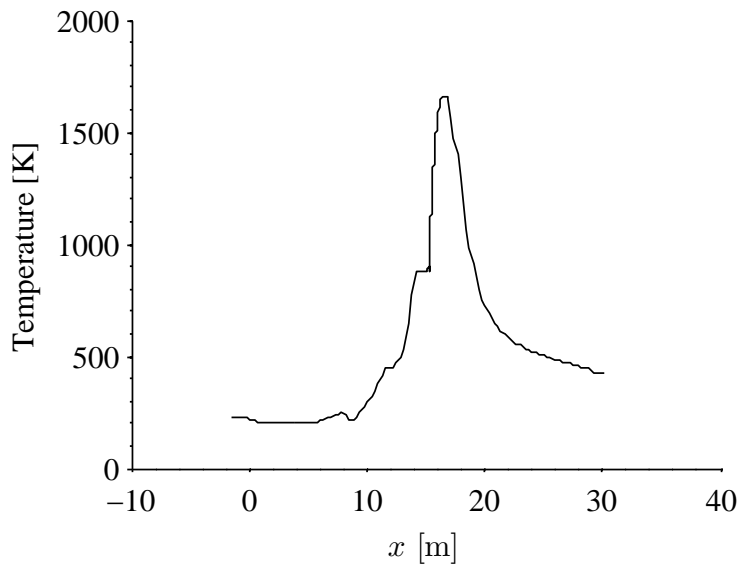

b) Temperature.

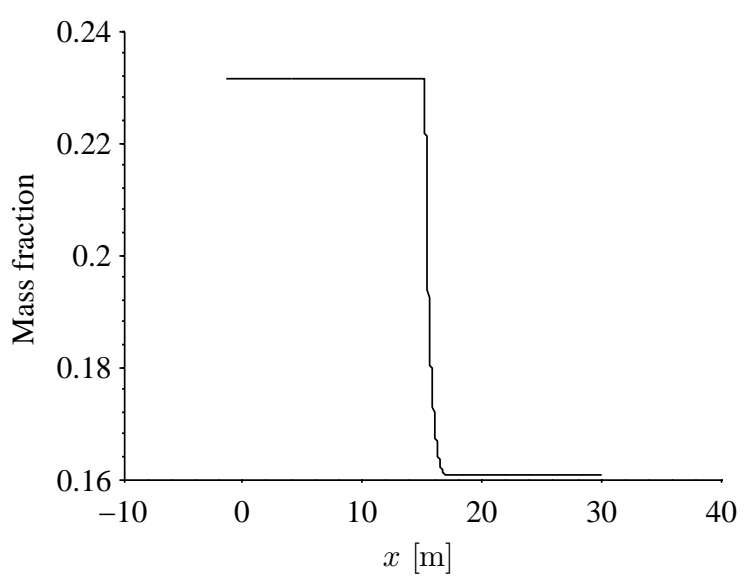

d) Mass fraction of $\mathrm{O}_{2}$.

Figure 4. One-dimensional profiles for trimmed flight condition at Mach 8 and an altitude of $26 \mathrm{~km}$.

specific moment in the body frame, which we write

$$
\begin{aligned}
\mathbf{f}^{b} & =\mathbf{f}^{b}(\mathbf{x}, \mathbf{u}) \\
\mathbf{M}^{b} & =\mathbf{M}^{b}(\mathbf{x}, \mathbf{u})
\end{aligned}
$$

Here $\mathbf{x}$ is a vector of state variables, and $\mathbf{u}$ is a vector of control variables. Since these forces and moments are calculated in a frame fixed to the body of the vehicle, we will write the equations of motion in this coordinate system. Since this frame is not an inertial frame, we cannot calculate the accelerations by just adding up all the forces in the body frame and dividing by the mass. Instead, we must apply the Coriolis transport theorem to account for the rotation of the body-fixed frame.

\section{A. Coordinate Systems}

In order to apply Newton's second law directly, we need an inertial frame. For our purposes, this will be the Earthcentered inertial (ECI) frame, and we label it the $i$-frame.

$$
\ddot{\mathbf{r}}_{i b}^{i}=\mathbf{f}^{i}+\mathbf{g}^{i}
$$

We also see the need to be able to transform a vector from one coordinate system to another. We do this with a transformation matrix, for which we use the following notation.

$$
\mathbf{f}^{i}=\mathbf{C}_{b}^{i} \mathbf{f}^{b}
$$

$$
6 \text { of } 19
$$


Here the $b$ denotes the body frame, which is attached to the vehicle such that the $x$-axis points forward, the $y$-axis points to the right, and the $z$-axis points downward. This notation is saying that multiplying a vector that is resolved in the $b$-frame by $\mathbf{C}_{b}^{i}$ on the left results in the same vector resolved in the $i$-frame.

The notation $\mathbf{r}_{i b}^{i}$ requires more explanation. The $\mathbf{r}_{i b}$ part refers to the vector from the origin of the $i$-frame (the center of the Earth) to the origin of the $b$-frame (the center of mass in our case). The superscript $i$ means that the vector is written as a vector in the $i$ coordinate system.

Since the Earth is rotating, and the atmosphere more or less rotates with it (neglecting weather patterns), we describe the motion of the vehicle in a coordinate system where each point on the surface of the Earth has constant coordinates. For this purpose, we use the Earth-centered, Earth-fixed frame (ECEF), in which the $z^{e}$-axis points from the center of the Earth toward the north pole, the $x^{e}$-axis points toward the intersection of the equator and the prime meridian, and the $y^{e}$-axis completes a right-handed system. The angular velocity between the ECEF frame and the ECI frame is

$$
\boldsymbol{\omega}_{i e}^{e}=\left[\begin{array}{c}
0 \\
0 \\
\omega_{i e}
\end{array}\right] \quad \omega_{i e} \approx 7.292115 \times 10^{-5} \mathrm{rad} / \mathrm{s}
$$

To make the equations more intuitive, we introduce a coordinate system in which the $x$-axis points toward the local north, the $y$-axis points toward the local east, and the $z$-axis points locally down. This is called the navigation frame, and the transformation matrix from the navigation frame to the ECEF frame is

$$
\mathbf{C}_{n}^{e}=\left[\begin{array}{ccc}
-\sin L \cos \lambda & -\sin \lambda & -\cos L \cos \lambda \\
-\sin L \sin \lambda & \cos \lambda & -\cos L \sin \lambda \\
\cos L & 0 & -\sin L
\end{array}\right]
$$

The navigation frame is carried with the vehicle, so that the origin of the navigation frame is always located at the center of mass of the vehicle. However, the navigation frame does not rotate with the vehicle, so that, for example, the $z^{n}$-axis always points directly normal to the surface of the Earth. Figure 5 gives a visual demonstration of the relation between the ECEF and navigation coordinate frames for an example point. The components of the vehicle velocity in this frame are

$$
\mathbf{v}_{e b}^{n}=\mathbf{C}_{e}^{n} \dot{\mathbf{r}}_{e b}^{e}=\left[\begin{array}{l}
v_{N} \\
v_{E} \\
v_{D}
\end{array}\right]
$$

This equation shows an implicit assumption made in this notation; derivatives that are shown with a dot are always taken in the resolving frame. This means, for example, that $\mathbf{v}_{e b}^{n} \neq \dot{\mathbf{r}}_{e b}^{n}$. Also, the vehicle velocity and position are both always measured from the ECEF origin to the body origin, so the two subscripts will be dropped in the following references.

The final coordinate frame is the body frame, which was discussed previously and has the transformation matrix that carries the navigation frame to the body frame of

$$
\mathbf{C}_{n}^{b}=\left[\begin{array}{ccc}
1 & 0 & 0 \\
0 & \cos \phi & \sin \phi \\
0 & -\sin \phi & \cos \phi
\end{array}\right]\left[\begin{array}{ccc}
\cos \theta & 0 & -\sin \theta \\
0 & 1 & 0 \\
\sin \theta & 0 & \cos \theta
\end{array}\right]\left[\begin{array}{ccc}
\cos \psi & \sin \psi & 0 \\
-\sin \psi & \cos \psi & 0 \\
0 & 0 & 1
\end{array}\right]
$$

The components of the velocity are

$$
\mathbf{v}^{b}=\mathbf{C}_{e}^{b} \dot{\mathbf{r}}^{e}=\left[\begin{array}{c}
u \\
v \\
w
\end{array}\right]
$$

\section{B. The WGS84 Earth Model}

Instead of using a spherical Earth, we have chosen to use the WGS84 ellipsoidal reference datum [22]. This is the same model used for the Global Positioning System (GPS) [23], and it thus provides an accurate reference for navigation. In this model, each curve of constant latitude is a circle, but each meridian is an ellipse with an eccentricity of

$$
e=0.0818191908426
$$

and a semi-major axis of

$$
R_{Q}=6378137 \mathrm{~m}
$$




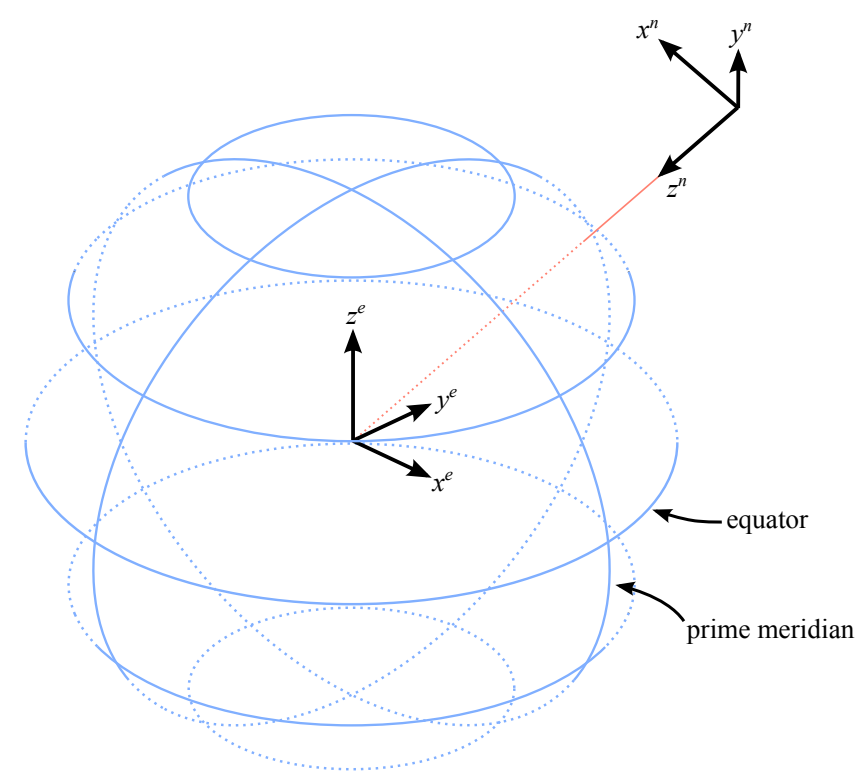

Figure 5. ECEF and navigation coordinate frames

One result of using an ellipsoidal Earth is the possibility of ambiguously defined latitude and altitude. In this paper, we use geodetic latitude, which has the property that moving normal to the reference ellipsoid does not change the latitude. As a result, the ECEF coordinates in terms of latitude, longitude, and altitude are

$$
\begin{aligned}
x_{e n}^{e} & =\left(\frac{R_{Q}}{\sqrt{1-e^{2} \sin ^{2} L}}+h\right) \cos L \cos \lambda \\
y_{e n}^{e} & =\left(\frac{R_{Q}}{\sqrt{1-e^{2} \sin ^{2} L}}+h\right) \cos L \sin \lambda \\
z_{e n}^{e} & =\left(\frac{R_{Q}\left(1-e^{2}\right)}{\sqrt{1-e^{2} \sin ^{2} L}}+h\right) \sin L
\end{aligned}
$$

We also need the rotation rate and the angular acceleration of the $n$-frame with respect to the $e$-frame, which are

$$
\boldsymbol{\omega}_{e n}^{n}=\left[\begin{array}{c}
\dot{\lambda} \cos L \\
-\dot{L} \\
-\dot{\lambda} \sin L
\end{array}\right] \quad \dot{\boldsymbol{\omega}}_{e n}^{n}=\left[\begin{array}{c}
\ddot{\lambda} \cos L-\dot{\lambda} \dot{L} \sin L \\
-\ddot{L} \\
-\ddot{\lambda} \sin L-\dot{\lambda} \dot{L} \cos L
\end{array}\right]
$$

where

$$
\begin{array}{ll}
\dot{\lambda}=\frac{v_{E}}{R_{E}} & \ddot{\lambda}=\left(\frac{\dot{v}_{E}}{v_{E}}-\frac{\dot{R}_{E}}{R_{E}}\right) \dot{\lambda} \\
\dot{L}=\frac{v_{N}}{R_{N}} & \ddot{L}=\left(\frac{\dot{v}_{N}}{v_{N}}-\frac{\dot{R}_{N}}{R_{N}}\right) \dot{L}
\end{array}
$$

Here $r_{E}$ refers to the radius of curvature in the east-west direction and $r_{N}$ is the radius of curvature in the north-south direction. Their formulas are

$$
\begin{array}{ll}
R_{E}=\left(\frac{R_{Q}}{\left.\sqrt{1-e^{2} \sin ^{2} L}+h\right) \cos L}\right. & \dot{R}_{E}=-v_{N} \sin L-v_{D} \cos L \\
R_{N}=\frac{R_{Q}\left(1-e^{2}\right)}{\left(1-e^{2} \sin ^{2} L\right)^{3 / 2}}+h & \dot{R}_{N}=\frac{3}{2} \frac{\left(R_{N}-h\right) e^{2} \sin 2 L}{1-e^{2} \sin ^{2} L} \frac{v_{N}}{R_{N}}-v_{D}
\end{array}
$$




\section{Net Accelerations}

The equations of motion in the $n$-frame, which are often called the navigation equations [23] are

$$
\dot{\mathbf{v}}^{n}=\left[\begin{array}{c}
\dot{v}_{N} \\
\dot{v}_{E} \\
\dot{v}_{D}
\end{array}\right]=\mathbf{C}_{b}^{n} \mathbf{f}^{b}+\mathbf{g}^{n}-\boldsymbol{\omega}_{i e}^{n} \times\left(\boldsymbol{\omega}_{i e}^{n} \times \mathbf{C}_{e}^{n} \mathbf{r}^{e}\right)-\left(2 \boldsymbol{\omega}_{i e}^{n}+\boldsymbol{\omega}_{e n}^{n}\right) \times \mathbf{v}^{n}
$$

A simple application of the transport theorem provides the net accelerations in the body frame.

$$
\dot{\mathbf{v}}^{b}=\left[\begin{array}{c}
\dot{u} \\
\dot{v} \\
\dot{w}
\end{array}\right]=\mathbf{f}^{b}+\mathbf{C}_{n}^{b}\left(\mathbf{g}^{n}-\boldsymbol{\omega}_{i e}^{n} \times\left(\boldsymbol{\omega}_{i e}^{n} \times \mathbf{C}_{e}^{n} \mathbf{r}^{e}\right)-\left(2 \boldsymbol{\omega}_{i e}^{n}+\boldsymbol{\omega}_{e n}^{n}+\mathbf{C}_{b}^{n} \boldsymbol{\omega}_{n b}^{b}\right) \times \mathbf{v}^{n}\right)
$$

The angular equations of motion are derived in a similar fashion. The resulting equations are

$$
\dot{\boldsymbol{\omega}}_{n b}^{b}=\left(\mathbf{I}^{b}\right)^{-1}\left(\mathbf{M}^{b}-\boldsymbol{\omega}_{i b}^{b} \times \mathbf{I}^{b} \boldsymbol{\omega}_{i b}^{b}\right)-\mathbf{C}_{n}^{b}\left(\dot{\boldsymbol{\omega}}_{e n}^{n}+\boldsymbol{\omega}_{i n}^{n} \times \mathbf{C}_{b}^{n} \boldsymbol{\omega}_{n b}^{b}+\boldsymbol{\omega}_{i e}^{n} \times \boldsymbol{\omega}_{e n}^{n}\right)
$$

where

$$
\begin{aligned}
\boldsymbol{\omega}_{i n}^{n} & =\boldsymbol{\omega}_{i e}^{n}+\boldsymbol{\omega}_{e n}^{n} \\
\boldsymbol{\omega}_{i b}^{b} & =\mathbf{C}_{n}^{b} \boldsymbol{\omega}_{i n}^{n}+\boldsymbol{\omega}_{n b}^{b}
\end{aligned}
$$

and $\mathbf{I}^{b}$ is the inertia tensor divided by the total mass of the vehicle. In our model, we assume that changes in the mass of the vehicle simply change the uniform density of the entire vehicle volume, which is a first approximation used before the mass properties of the vehicle are realistically established. As a result, $\mathbf{I}^{b}$ is independent of the vehicle mass in this simple model. The components of the body angular velocity and its derivative are

$$
\boldsymbol{\omega}_{n b}^{b}=\left[\begin{array}{c}
p \\
q \\
r
\end{array}\right] \quad \dot{\boldsymbol{\omega}}_{n b}^{b}=\left[\begin{array}{c}
\dot{p} \\
\dot{q} \\
\dot{r}
\end{array}\right]
$$

\section{Trim}

For the purposes of finding a trimmed flight condition, we combine the equations of motion into the form

$$
\mathbf{y}=\mathbf{F}(\mathbf{x}, \mathbf{u})
$$

The output vector, $\mathbf{y}$, consists of the velocity and angular velocity derivatives, $\dot{u}, \dot{v}, \dot{w}, \dot{p}, \dot{q}$, and $\dot{r}$. Finding a trimmed flight condition, then, reduces to picking a value of $\mathbf{y}$ (usually $\mathbf{0}$ ), and finding values of $\mathbf{x}$ and $\mathbf{u}$ that satisfy Eq. (22). Typically, we want to select values for some of the state variables and find values for the remaining state variables and the control variables. Using these concepts, we can rewrite Eq. (22) as

$$
\mathbf{y}=\mathbf{F}(\boldsymbol{\xi}, \boldsymbol{v})
$$

where $\boldsymbol{\xi}$ is a vector of the independent state variables, and $\boldsymbol{v}$ contains the dependent state variables and the control variables. In this discussion, we consider the independent state variables to be those describing the position, velocity, and angular velocity. The dependent variables include the orientation of the vehicle and the various control variables.

For this paper, the independent variables are

$$
\boldsymbol{\xi}=\left[\begin{array}{lllllllll}
L & \lambda & h & M & \gamma & \sigma & p & q & r
\end{array}\right]^{T}
$$

and the dependent variables are

$$
\boldsymbol{v}=\left[\begin{array}{llllllll}
\alpha & \beta & \phi & \delta_{E R} & \delta_{C E} & \delta_{D E} & \delta_{C R} & \delta_{D R}
\end{array}\right]^{T}
$$

where $\delta_{C E}$ is the average of the left and right elevator deflection angles (defined so that a positive deflection moves the trailing edge down) and $\delta_{D E}$ is the deflection angle of the right elevator minus the deflection angle of the left elevator. For simplicity we also make the assumption that the sideslip angle, $\beta$, and the differential rudder angle, $\delta_{D R}$, are both zero. This is convenient because it makes Eq. (23) a system of equations with six variables and six equations.

In our case, evaluating the function $\mathbf{F}$ is relatively expensive. As a result, we want to use a gradient-based method, but we also would not like to have to evaluate the function seven times at each iteration. To alleviate this, we use Broyden's method [24], which requires seven function evaluations to calculate the first estimate of the gradient matrix, $\partial \mathbf{F} / \partial \boldsymbol{v}$. Subsequent estimates are obtained using only the previous estimate of the gradient and the function evaluation of the current iteration. 


\section{E. Turning}

Using the traditional flat-Earth equations of motion, a $2 g$-turn refers to a maneuver in which the pilot experiences a force that is twice as strong as gravity. In this case we would write $n=\left\|\mathbf{f}^{b}\right\| / g$ where the load factor $n$ would be 2 for a $2 g$-turn. We could use the same definition for hypersonic flight, but it would give the unusual result that a $1 g$-turn would still have some component of turning. Instead we define the turning coefficient so that the component of the acceleration normal to the velocity is the same as it would be for the flat-Earth equations. Thus for a vehicle that is flying directly eastward, the acceleration to the north is given by

$$
\dot{v}_{N}=g \sqrt{n^{2}-1}
$$

\section{F. Linearization}

We can write our equations of motion as a generic nonlinear system

$$
\dot{\mathbf{x}}=\mathbf{F}(\mathbf{x}, \mathbf{u})
$$

For small perturbations from a trimmed flight condition, it is useful to study a linearized system of equations. To do this, we write

$$
\mathbf{x}=\overline{\mathbf{x}}+\Delta \mathbf{x} \quad \mathbf{u}=\overline{\mathbf{u}}+\Delta \mathbf{u}
$$

and substitute this into the original nonlinear system, Eq. (27). After taking a first-order Taylor series and canceling the trimmed part of the equations of motion, we get

$$
\Delta \dot{\mathbf{x}} \approx \mathbf{A} \Delta \mathbf{x}+\mathbf{B} \Delta \mathbf{u}
$$

Analysis of the linearized dynamics gives insight into the stability and control characteristics of the vehicle. The eigenvalues of $\mathbf{A}$ give the open-loop stability characteristics of the trimmed flight condition, and by selecting the controlled states and the corresponding control effectors to be used, we can also calculate the transmission zeros of the system.

The MASIV vehicle model often predicts that the thrust of the vehicle is not a smooth function of angle of attack and Mach number [9]. Although the deviation from smoothness is only visible at small scales, this creates some difficulties in the estimation of the derivative matrices $\partial \mathbf{F} / \partial \mathbf{x}$ and $\partial \mathbf{F} / \partial \mathbf{u}$. Since these small-scale deviations are probably not physical, we chose to employ a method that reduces noise when calculating the derivative matrices. Rather than using a centered difference to calculate each derivative,

$$
\frac{\partial F_{j}}{\partial x_{k}} \approx \frac{F_{j}\left(\overline{\mathbf{x}}+\eta_{k} \mathbf{e}_{k}, \overline{\mathbf{u}}\right)-F_{j}\left(\overline{\mathbf{x}}-\eta_{k} \mathbf{e}_{k}, \overline{\mathbf{u}}\right)}{2 \eta_{k}}
$$

we use extra function evaluations (i.e. more than twice the number of state variables and control variables) and then fit a plane to the data using the method of linear least squares. This technique is essentially a low-pass filter, which reduces high-frequency noise. The idea was formalized by Savitzky and Golay [25], although it has been around since the $19^{\text {th }}$ century [26].

\section{Results}

The primary result of this work is a set of operating maps for both turning and straight flight. Each operating map is essentially a plot of the nonzero values of $v$ for a range of flight conditions. The second part of the results discusses the properties of the linearized equations of motion at a selected turning flight condition.

In this paper, all results are for the vehicle shown in Figs. 1 and 2. The vehicle has two all-moving horizontal surfaces, which we refer to as elevators. There are also two rudders, although we do not allow them to move independently in these results. The inertia properties of the vehicle are given in Table 1, and the fuel tanks are set to half full for all cases. The mass estimates come from several correlations, which are given in [27]. The inertia tensor is calculated by assuming a constant density throughout the vehicle.

Figure 6 shows an operating map for level, unaccelerated flight eastward over the equator. In other words, $L=0$, $\gamma=0, \sigma=90^{\circ}$, and $n=1$. In this case, the flight is longitudinal, so there are only three nonzero trim values. Figure 7 shows an operating map for the same conditions except with $n=2$, which corresponds to a vehicle that is initially flying to the east but with a southward lateral acceleration of $17 \mathrm{~m} / \mathrm{s}^{2}$. For the turning conditions, the roll angle varies between $62^{\circ}$ and $66^{\circ}$. Since $n$ is constant in Fig. 7, the bank angle (which is similar to the roll angle) might 


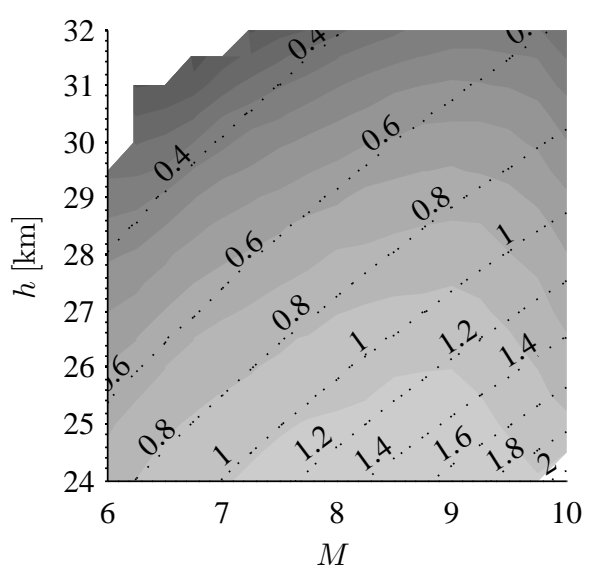

a) Equivalence ratio, $\delta_{E R}$.

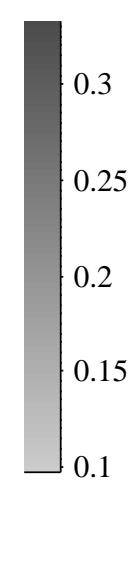

0.25

2

0.15

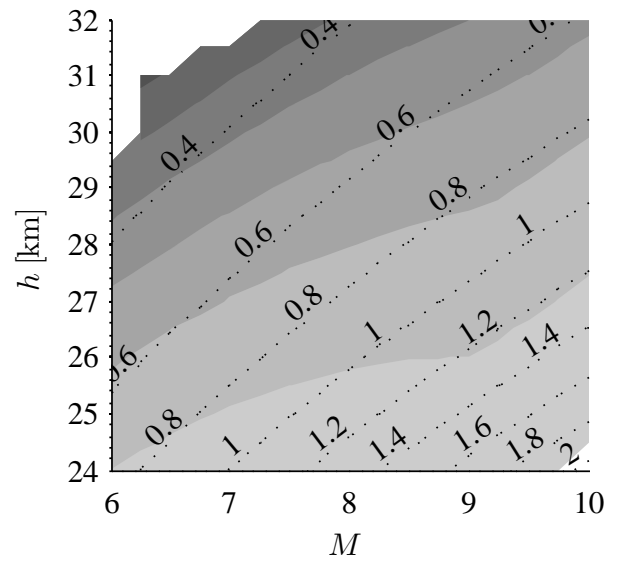

b) Angle of attack, $\alpha$ (degrees).

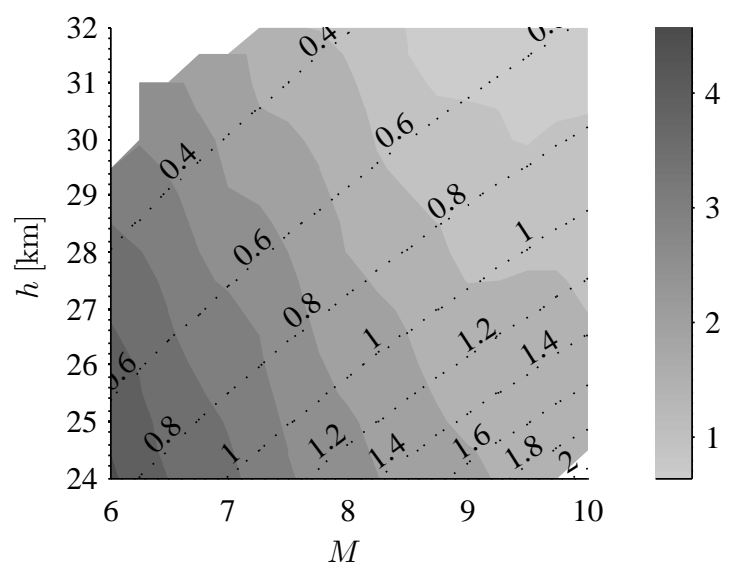

c) Elevator angle, $\delta_{C E}$ (degrees).

Figure 6. Operating maps for steady, level flight for the half-fueled vehicle flying eastward over the equator. Dotted lines show contours of dynamic pressure in atmospheres. 


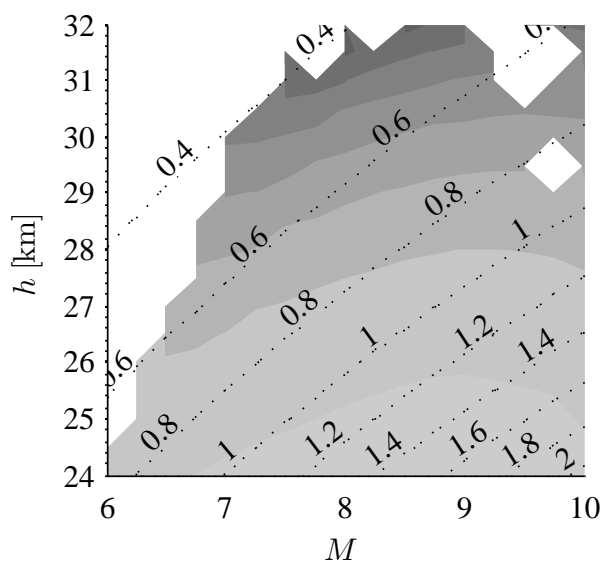

a) Equivalence ratio, $\delta_{E R}$.

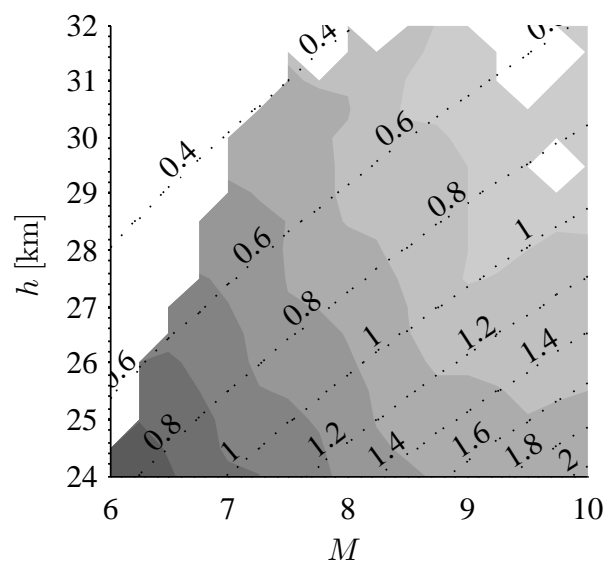

c) Elevator angle, $\delta_{C E}$ (degrees).
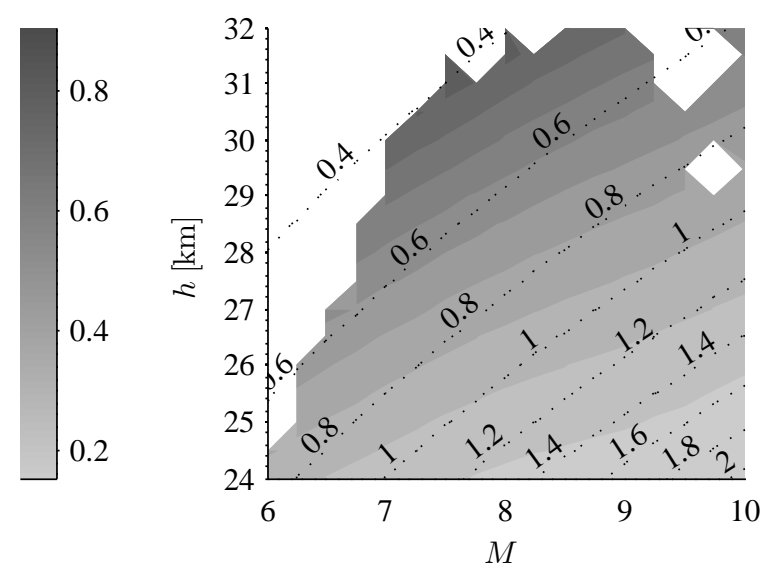

b) Angle of attack, $\alpha$ (degrees).
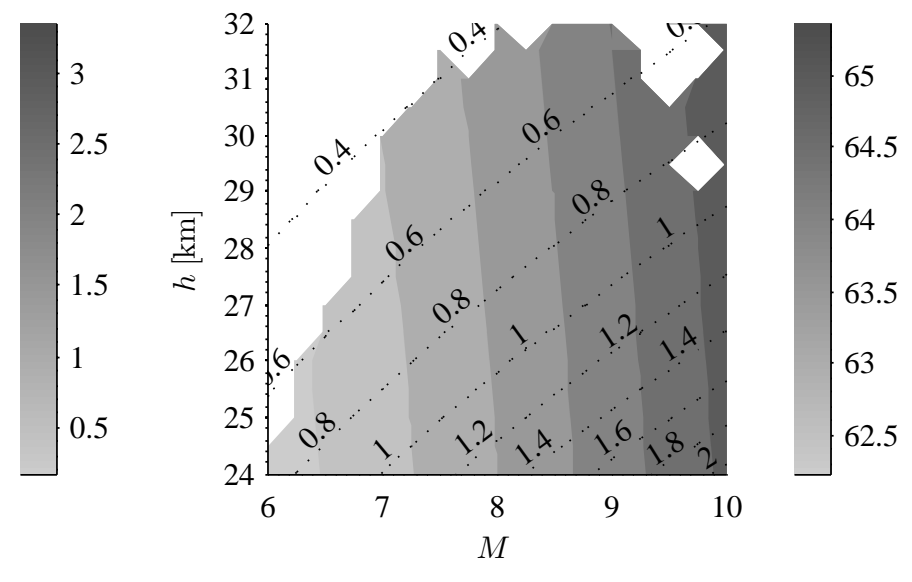

d) Roll angle, $\phi$ (degrees).

Figure 7. Operating maps for turning flight starting from eastward flight over the equator. Dotted lines show contours of dynamic pressure in atmospheres. 
Table 1. Inertia properties of example vehicle.

\begin{tabular}{ccc}
\hline \hline Symbol & Name & Value \\
\hline & operational empty mass & $1.90 \times 10^{4} \mathrm{~kg}$ \\
& maximum fuel mass & $2.27 \times 10^{4} \mathrm{~kg}$ \\
$I_{x x}$ & specific moment of inertia & $2.45 \mathrm{~m}^{2}$ \\
$I_{y y}$ & specific moment of inertia & $34.93 \mathrm{~m}^{2}$ \\
$I_{z z}$ & specific moment of inertia & $36.34 \mathrm{~m}^{2}$ \\
$I_{x z}$ & specific product of inertia & $0.50 \mathrm{~m}^{2}$ \\
\hline \hline
\end{tabular}

be expected to be constant. However, the roll angle, which is shown in Fig. 7d, varies somewhat with Mach number because of the definition used for $n$.

In each plot of Figs. 6 and 7, lines of constant dynamic pressure are plotted on top of the operating map. The values associated with each dynamic pressure contour are given in atmospheres. These lines of constant dynamic pressure serve two purposes: to show where lines of constant dynamic pressure are on the operating map and to suggest an upper limit on the Mach number for each altitude. The model used for these operating maps does not include an analysis of heat transfer, so the model predicts that the vehicle is able to trim at very high Mach numbers. However, a possible first approximation to the effect of heating considerations on the flight envelope is to limit the dynamic pressure to about 1 atmosphere.

In both of the operating maps, many of the same trends are present. The white area on the left of each plot shows the area in which the engine operates in ram mode. Ram mode occurs when MASIV predicts that the local Mach number inside the combustor approaches one at some point. The transition to ram mode is essentially the lower Mach number limit of the scram-mode flight envelope. Notably, the boundary between ram mode and scram mode is at a higher Mach number for the turning case. This is not surprising because the turn requires more lift, and thus a higher angle of attack. At a higher angle of attack, the vehicle will have more drag, and thus more fuel is needed. The higher equivalence ratio can be seen in the comparison between Figs. 6a and 7a.

The trends in equivalence ratio and angle of attack are also very interesting. If we increase the Mach number while holding the altitude constant, the vehicle naturally gets more lift, so the trimmed angle of attack decreases. At the same time, the drag increases, so the more thrust is needed to trim the acceleration to zero. However, the mass flow rate also increases with Mach number, and the result is that the trimmed equivalence ratio actually decreases until the Mach number reaches about 9. The inlet used for this vehicle was designed to operate for Mach numbers between 7 and 9 using the methodology outlined in [10], and we see sharp changes in the operating maps near Mach 9. For higher Mach numbers, the inlet behaves poorly due to increased shock wave interactions, and the drag increases very rapidly. The result is that a large amount of fuel is needed for flight at Mach numbers greater than 9 .

In Fig. 8, a similar set of plots is presented, except that the dynamic pressure is held constant at $1 \mathrm{~atm}$ so that the altitude is a function of the Mach number. The result is an operating map for various strengths of turn at each Mach number. The routine is able to find trimmed flight conditions for all combinations of Mach number and turn strength. Contrast this with Figs. 6 and 7, in which a significant portion of the flight conditions could not be trimmed. Actually, this should not be a surprise considering that the vehicle was able to trim along the contour where the dynamic pressure is $1 \mathrm{~atm}$ in both figures.

Figure 8a shows that the trimmed equivalence ratio must increase as a function of both $M$ and $n$. The trend of $\delta_{E R}$ with $n$ is not surprising considering that the vehicle is using essentially an increase in lift to execute the turn. The trend of $\delta_{E R}$ as a function of $M$ is slightly more interesting because an increase in Mach number along a trajectory with constant dynamic pressure has the opposite effect on $\delta_{E R}$ as an increase in $M$ at a constant altitude. In other words, a flat acceleration and a climbing acceleration require opposite changes to the thrust setting. The explanation for the flat acceleration was given in a previous paragraph, and the climbing result has a very similar explanation. If the dynamic pressure $\left(\rho u^{2}\right)$ is constant and the Mach number (and thus $u$ ) increases, then the mass flow rate ( $\rho u$ ) must decrease. Thus if the drag remains about the same, we would expect a higher required equivalence ratio to produce the same thrust with a lower air mass flow. This analysis relies on a number of simplifications and assumptions, so it is not exactly the type of logic that should be used to predict the behavior of $\delta_{E R}$ as a function of $M$, but it does seem to offer a plausible explanation of the trends visible in Fig. 6a.

The angle of attack in Fig. $8 \mathrm{~b}$ tends to increase with the Mach number. The increase is slow (about $0.25^{\circ}$ for every integer increase in Mach number) until the Mach number reaches 9. At higher speeds, the angle of attack is approximately constant. The plot also shows that the angle of attack increases as a function of $n$, which is quite 


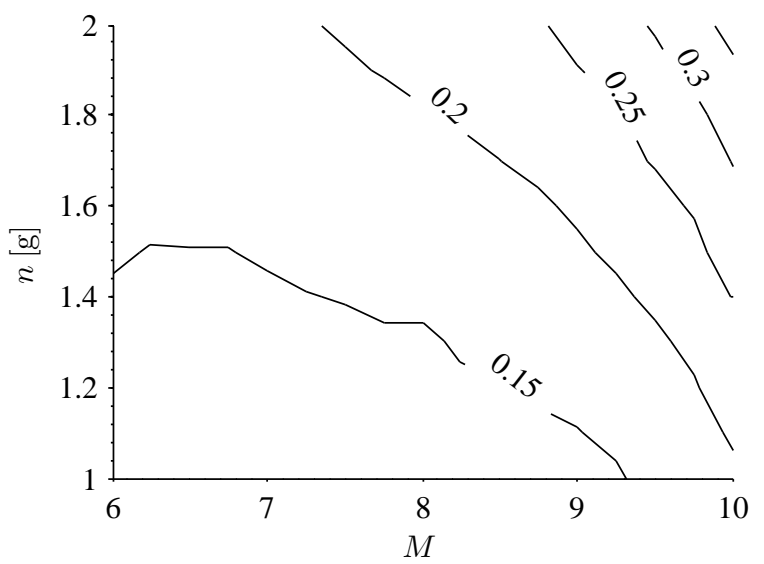

a) Equivalence ratio, $\delta_{E R}$.

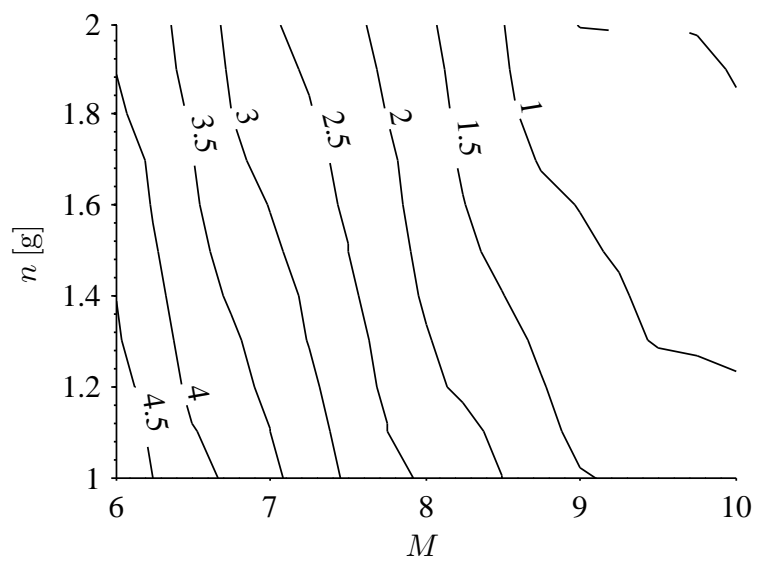

c) Elevator angle, $\delta_{C E}$.

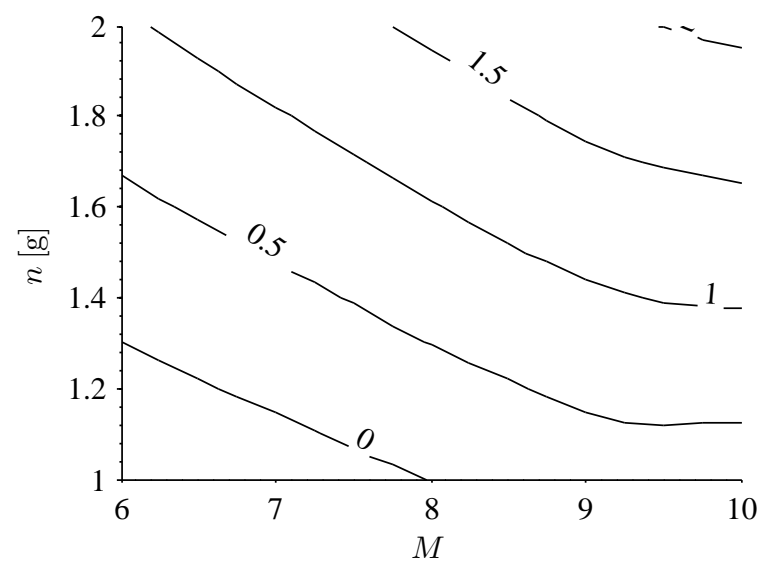

b) Angle of attack, $\alpha$ (degrees).

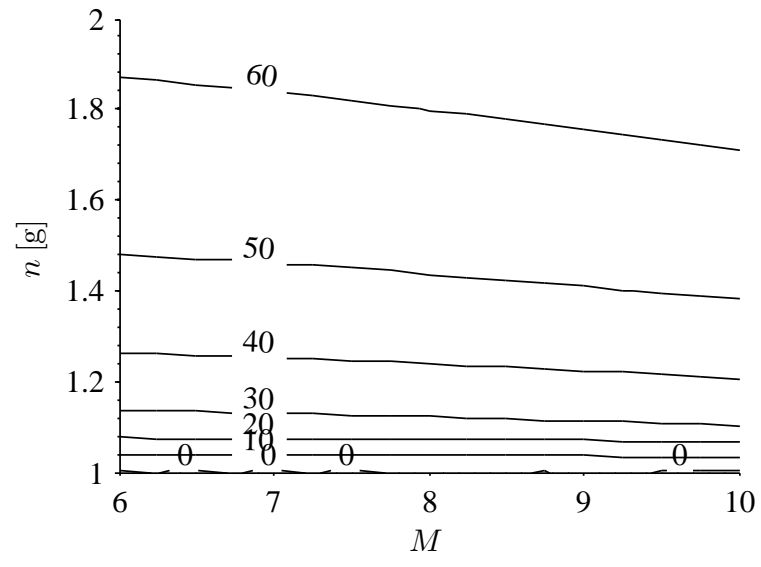

d) Roll angle, $\phi$ (degrees).

Figure 8. Operating map for various turning conditions. The dynamic pressure is constant at 1 atm. 
obvious given that the vehicle uses extra lift to turn. For a vehicle that must operate over a large range of Mach numbers (especially as part of a launch vehicle), it would be ideal if the trimmed angle of attack is a weak function of Mach number. The trends in Fig. 6b, namely that angle of attack decreases with altitude and decreases with Mach number, indicate that it may be possible to design a vehicle that has a constant angle of attack along a climbing and accelerating trajectory.

The plot in Fig. 8c shows that the elevator setting changes very little as a function of the load factor, but it increases relatively rapidly with Mach number. Like most of the other trends, the behavior changes for Mach numbers greater than 9. From these plots, we can see that the vehicle has a reasonable ability to turn, and that further optimization of the vehicle could give a much larger flight envelope.

\section{A. Linearized Motion Properties}

We performed a linearization of the equations of motion at two flight conditions. Both flight conditions are for the half-fueled vehicle flying eastward over the equator with a dynamic pressure of $1 \mathrm{~atm}$. The first case, for which the pole/transmission zero maps are shown in Fig. 9, is for unturning flight while Fig. 10 shows the maps for turning flight. The two flight conditions, about which the linear systems are obtained, are tabulated in Table 2.

Table 2. Trim conditions for the linearizations.

\begin{tabular}{ccc}
\hline \hline State or input & Fig. $9, n=0$ & Fig. $10, n=2$ \\
\hline$h$ & $26 \mathrm{~km}$ & $26 \mathrm{~km}$ \\
$V$ & $2394 \mathrm{~m} / \mathrm{s}$ & $2394 \mathrm{~m} / \mathrm{s}$ \\
$\alpha$ & $0.046^{\circ}$ & $1.67^{\circ}$ \\
$\beta$ & $0^{\circ}$ & $0^{\circ}$ \\
$\phi$ & $0^{\circ}$ & $63.54^{\circ}$ \\
$\psi$ & $90^{\circ}$ & $91.5^{\circ}$ \\
$\theta$ & $0.046^{\circ}$ & $0.74^{\circ}$ \\
$\delta_{E R}$ & 0.127 & 0.227 \\
$\delta_{C E}$ & $2.40^{\circ}$ & $1.56^{\circ}$ \\
$\delta_{D E}$ & $0^{\circ}$ & $0^{\circ}$ \\
$\delta_{D R}$ & $0^{\circ}$ & $0^{\circ}$ \\
\hline \hline
\end{tabular}

We used

$$
\mathbf{x}=\left[\begin{array}{llllllllll}
h & V & \alpha & \beta & \phi & \theta & \psi & p & q & r
\end{array}\right]^{T}
$$

as the full set of state variables, and the full set of inputs was

$$
\mathbf{u}=\left[\begin{array}{llll}
\delta_{E R} & \delta_{C E} & \delta_{D E} & \delta_{C R}
\end{array}\right]^{T}
$$

Finally, the outputs were selected to be

$$
\mathbf{y}=\left[\begin{array}{llll}
V & \gamma & \sigma & \phi
\end{array}\right]^{T}
$$

The states were split into longitudinal states of $h, V, \alpha, \theta$, and $q$ and lateral states of $\beta, \phi, \psi, p$, and $r$. The longitudinal inputs are $\delta_{E R}$ and $\delta_{C E}$, and the lateral inputs are $\delta_{D E}$ and $\delta_{C R}$. Finally, the outputs were split into longitudinal outputs, $V$ and $\gamma$, and lateral outputs, $\chi$ and $\phi$.

The longitudinal dynamics, shown in Figs. 9a and 10a, are quite similar between the two cases while the lateral dynamics, shown in Figs. 9a and 10a, show more notable differences. In both cases, the poles are quite similar, but the zeros have moved significantly. For the $n=1$ case, both zeros are on the imaginary axis, while both are on the real axis for $n=2$. Actually, a zero at -735 is not shown in Fig. 10b because it would give the plot too large of a range of scales. The main reason for the difference in linearization zeros is that the roll state, $\phi$, is not zero in the turning case. The result is increased control authority of the heading output, $\sigma$. Since roll is the most effective way to control heading, the response of the system to small changes in roll is important in determining how easily $\sigma$ can be controlled. However, when $\phi$ is zero, small changes in $\phi$ result in only small changes in the lateral force.

For both configurations, one of the zeros of the pitch dynamics is non-minimum phase, which is consistent with the results in [4]. This is expected because of the actions the vehicle needs to take to increase $\gamma$. In order to increase the climb rate, the vehicle needs to deflect the elevators upward to increase the angle of attack, but deflection the elevators decreases the lift instantaneously, which causes the aircraft to plunge and drop altitude before it begins to pitch nose-up 


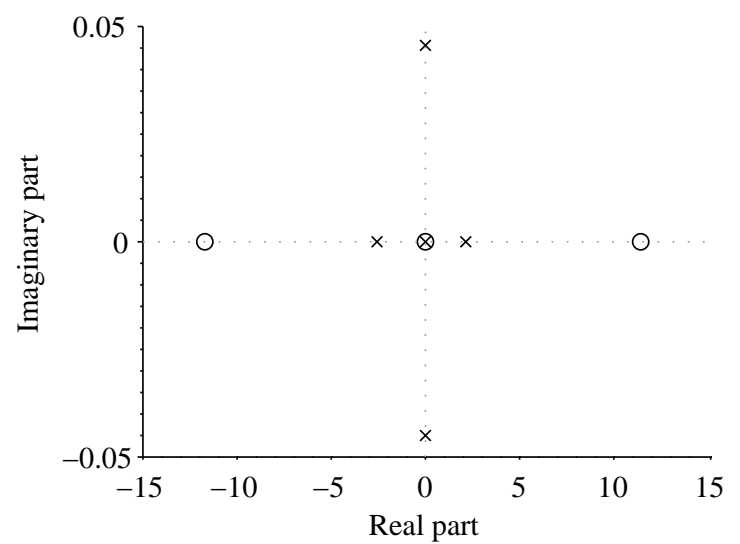

a) Longitudinal dynamics.

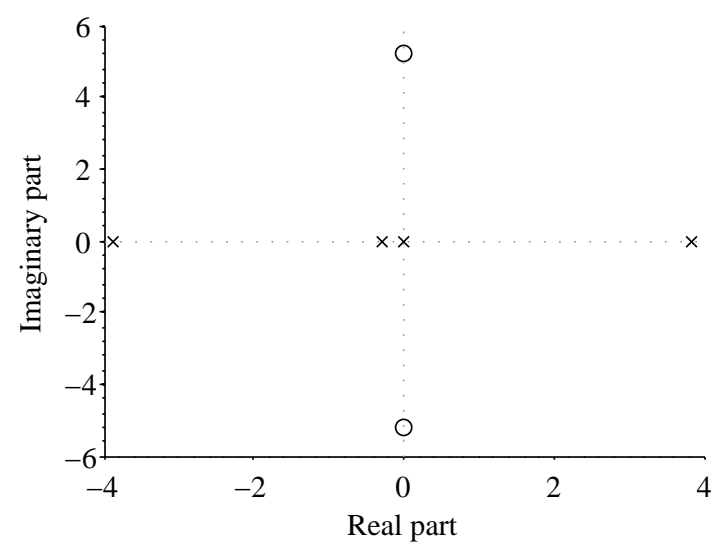

b) Lateral dynamics.

Figure 9. Pole/transmission zero maps of the Jacobian linearization about the unturning Mach 8 flight condition.

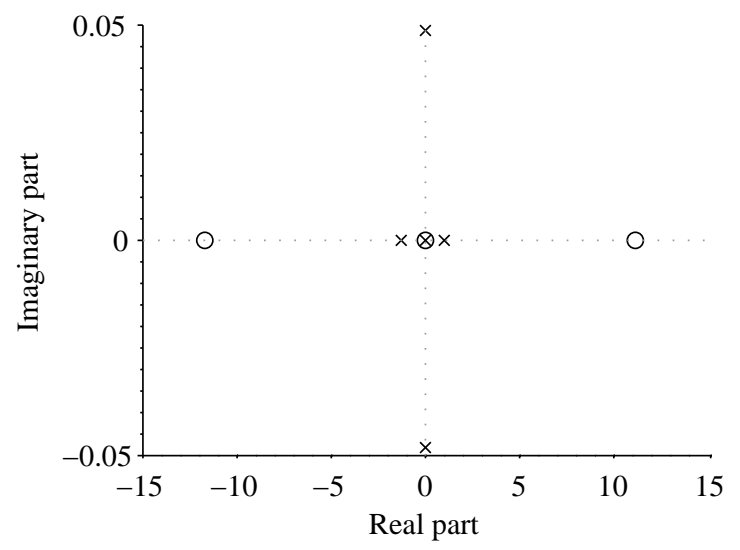

a) Longitudinal dynamics.

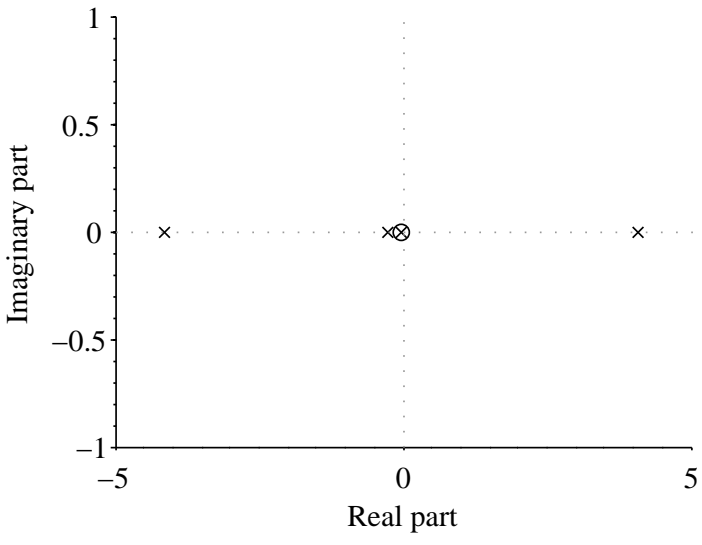

b) Lateral dynamics. A zero at -735 is not plotted.

Figure 10. Pole/transmission zero maps of the Jacobian linearization about the turning Mach 8 flight condition. 
and increase $\alpha$ and thus lift. These non-minimum phase dynamics affect the control design, and are important in this case because the vehicle is open-loop unstable, and the transmission zeros effectively limit the amount of bandwidth in the system.

Table 3. Longitudinal real poles and time-to-double or half-amplitude.

a) No turning, $n=1$.

\begin{tabular}{cc}
\hline \hline Pole & Time to double \\
\hline-2.46 & 0.31 \\
2.07 & 0.36 \\
-0.0036 & 192.3 \\
\hline
\end{tabular}

b) Turning, $n=2$.

\begin{tabular}{cc}
\hline \hline Pole & Time to double \\
\hline 0.36 & 1.93 \\
\hline \hline
\end{tabular}

Table 4. Longitudinal complex-conjugate poles, damping ratios, and natural frequencies.

a) No turning, $n=1$.

\begin{tabular}{ccc}
\hline \hline Pole & Damping ratio & Natural frequency \\
\hline$-2.49 \times 10^{-4} \pm 4.54 \times 10^{-2} j$ & $5.49 \times 10^{-2}$ & $4.54 \times 10^{-2}$ \\
\hline \hline
\end{tabular}

b) Turning, $n=2$.

\begin{tabular}{ccc}
\hline \hline Pole & Damping ratio & Natural frequency \\
\hline$-3.52 \times 10^{-1} \pm 8.97 \times 10^{-2} j$ & 0.97 & $3.63 \times 10^{-1}$ \\
$-1.99 \times 10^{-2} \pm 3.52 \times 10^{-2} j$ & 0.49 & $4.04 \times 10^{-2}$ \\
\hline \hline
\end{tabular}

Table 5. Lateral-directional real poles and time-to-double or half-amplitude.

a) No turning, $n=1$.

\begin{tabular}{ccccc}
\hline \hline Pole & Time to double & & Pole & Time to double \\
\cline { 1 - 2 } 3.83 & 0.18 & & 4.11 & 0.17 \\
-3.87 & 0.18 & & -4.15 & 0.17 \\
-0.28 & 2.51 & & -0.28 & 2.52 \\
-0.0028 & 245.6 & & -0.0016 & 439.8 \\
\hline \hline
\end{tabular}

Tables 3, 4, and 5 give a detailed analysis of the poles from Figs. 9 and 10. Previous experience [6] indicates that the short-period mode is unstable for the $n=1$ case. Interestingly, the short-period mode has become stable for the turning configuration, which can be seen from the pair of values missing from Table $3 \mathrm{~b}$ that have migrated to $4 \mathrm{~b}$. Otherwise, the poles are quite similar between the $n=1$ and $n=2$ cases.

\section{Conclusion}

In this paper, we used a control-oriented model to analyze the turning capabilities of an air-breathing hypersonic vehicle. The model includes predictions for wave interactions, fuel mixing, and finite-rate chemistry. This framework has been integrated into a three-dimensional vehicle design and a rotating, ellipsoidal Earth model for the equations of motion. Using this combined model, we determined more than 700 trimmed flight conditions. The parameters that were varied in this investigation were Mach number, altitude, and load factor. We also investigated the linearized dynamics for two of these trimmed flight conditions.

The main analysis presented in this paper for the various trim conditions was operating maps showing the appropriate equivalence ratio, angle of attack, elevator angle, and roll angle for steady turning flight as a function of Mach number and altitude. These operating maps reveal important properties of the vehicle's performance, such as the Mach number at which ram-scram transition occurs and how the vehicle reacts to flying at a Mach number above the inlet design specifications. Furthermore, the vehicle was shown to have respectable performance for turns with a load factor of 2 or lower. This type of performance analysis, and the modest computational resources required to do it, provides a useful tool during the conceptual design phase of the vehicle design process. 


\section{Acknowledgments}

This research is funded by the Air Force Research Laboratory/Air Vehicles Directorate grant FA 8650-07-2-3744 for the Michigan/AFRL Collaborative Center in Control Sciences (Michael Bolender as technical monitor).

\section{References}

[1] Schöttle, U. M. and Hillesheimer, M., "Performance Optimization of an Airbreathing Launch Vehicle by a Sequential Trajectory Optimization and Vehicle Design Scheme," AIAA Guidance, Navigation and Control Conference, 1991, AIAA Paper 91-2655.

[2] Chavez, F. R. and Schmidt, D. K., "Analytical Aeropropulsive/Aeroelastic Hypersonic-Vehicle Model with Dynamic Analysis," Journal of Guidance, Control, and Dynamics, Vol. 17, No. 6, 1994, pp. 1308-1319.

[3] Bolender, M. A. and Doman, D. B., "Nonlinear Longitudinal Dynamical Model of an Air-Breathing Hypersonic Vehicle," Journal of Spacecraft and Rockets, Vol. 44, No. 2, 2007, pp. 374-387.

[4] Parker, J. T., Serrani, A., Yurkovich, S., Bolender, M. A., and Doman, D. B., "Control-Oriented Modeling of an Air-Breathing Hypersonic Vehicle,” Journal of Guidance, Control, and Dynamics, Vol. 30, No. 3, 2007, pp. 856-869.

[5] Frendreis, S. G. V. and Cesnik, C. E. S., "3D Simulation of a Flexible Hypersonic Vehicle," Atmospheric Flight Mechanics Conference \& Exhibit, 2010, AIAA Paper 2010-8229.

[6] Dalle, D. J., Torrez, S. M., Driscoll, J. F., and Bolender, M. A., "Flight Envelope Calculation of a Hypersonic Vehicle Usinga First Principles-Derived Model," 17th AIAA International Space Planes and Hypersonic Systems and Technologies Conference, 2011.

[7] Dalle, D. J., Fotia, M. L., and Driscoll, J. F., "Reduced-Order Modeling of Two-Dimensional Supersonic Flows with Applications to Scramjet Inlets," Journal of Propulsion and Power, Vol. 26, No. 3, 2010, pp. 545-555.

[8] Dalle, D. J., Frendreis, S. G. V., Driscoll, J. F., and Cesnik, C. E. S., "Hypersonic Vehicle Flight Dynamics with Coupled Aerodynamics and Reduced-order Propulsive Models," AIAA Atmospheric Flight Mechanics Conference \& Exhibit, 2010, AIAA Paper 2010-7930.

[9] Torrez, S. M., Driscoll, J. F., Dalle, D. J., Bolender, M. A., and Doman, D. B., "Hypersonic Vehicle Thrust Sensitivity to Angle of Attack and Mach Number," AIAA Atmospheric Flight Mechanics Conference, 2009, AIAA Paper 2009-6152.

[10] Torrez, S. M., Driscoll, J. F., Dalle, D. J., and Fotia, M. L., "Preliminary Design Methodology for Hypersonic Engine Flowpaths," 16th AIAA/DLR/DGLR International Space Planes and Hypersonic Systems and Technologies Conference, 2009, AIAA Paper 2009-7289.

[11] Tausche, M., Janovsky, R., Scheper, M., Apeldoorn, J., Monti, R., Savina, R., Fumo, M. D. S., Paterna, D., Sotto, E. D., ao Branco, J., and Molina, R., "PHOEBUS: A High Lift-over-Drag Vehicle for Earth Reentry," 16th AIAA/DLR/DGLR International Space Planes and Hypersonic Systems and Technologies Conference, 2009, AIAA Paper 2009-7411.

[12] Eisler, G. R. and Hull, D. G., “Optimal Descending, Hypersonic Turn to Heading,” Journal of Guidance, Vol. 10, No. 3, 1987, pp. 255-261.

[13] Roenneke, A. J. and Cornwell, P. J., "Trajectory Control for a Low-Lift Re-Entry Vehicle,” Journal of Guidance, Control, and Dynamics, Vol. 16, No. 5, 1993, pp. 927-933.

[14] Mooij, E., "Numerical Investigation of Model Reference Adaptive Control for Hypersonic Aircraft," Journal of Guidance, Control, and Dynamics, Vol. 24, No. 2, 2001, pp. 315-323.

[15] Raney, D. and Lallman, F., “Control Integration Concept for Hypersonic Cruise-Turn Maneuvers," Tech. Rep. TP 3136, NASA, 1992.

[16] Tarpley, C. and Lewis, M. J., "Stability Derivatives for a Hypersonic Caret-Wing Waverider," Journal of Aircraft, Vol. 32, No. 4, 1995, pp. 795-803.

[17] Hall, K. C., Thomas, J. P., and Dowell, E. H., "Proper Orthogonal Decomposition Technique for Transonic Unsteady Aerodynamic Flows," AIAA Journal, Vol. 38, No. 10, 2000, pp. 1853-1862.

[18] White, F. M., Viscous Fluid Flow, McGraw-Hill, 3rd ed., 2006.

[19] Van Driest, E. R., “The Problem of Aerodynamic Heating,” Aeronautical Engineering Review, Vol. 15, No. 10, 1956, pp. 2641.

[20] Torrez, S. M., Dalle, D. J., and Driscoll, J. F., "Design of Dual-Mode Hypersonic Vehicle Flow Paths Using a Reduced-Order Model," 17th AIAA International Space Planes and Hypersonic Systems and Technologies Conference, April 2011.

[21] Torrez, S. M., Driscoll, J. F., Ihme, M., and Fotia, M. L., "Reduced Order Modeling of Turbulent Reacting Flows With Application to Scramjets," Journal of Propulsion and Power, Vol. 27, No. 2, March-April 2011, pp. 371-382.

[22] Anon., "Department of Defense World Geodetic System 1984," Tech. Rep. TR8350.2, 3rd ed., National Imagery and Mapping Agency (now NGA), 1997. 
[23] Groves, P. D., Principles of GNSS, Inertial, and Multisensor Integrated Navigations Systems, Artec House, 2008.

[24] Broyden, C. G., "A Class of Methods for Solving Nonlinear Simultaneous Equations," Mathematics of Computation, Vol. 19, No. 92, October 1965, pp. 577-593.

[25] Savitzky, A. and Golay, M. J. E., "Smoothing and Differentiation of Data by Simplified Least Squares Procedures," Analytical Chemistry, Vol. 36, No. 8, 1964, pp. 1627-1639.

[26] Whittaker, S. E. and Robinson, G., The Calculus of Observations, Blackie \& Son, Limited, 1924.

[27] Chudoba, B., “Aircraft Volume and Mass Guidelines,” Tech. rep., National Institute of Aerospace, June 2008, Hypersonic Educational Initiative Hypersonic Vehicle System Integration Short Course. 